\title{
COVID-19 in long-term care facilities in South Africa: No time for complacency
}

COVID-19 cases and deaths continue to increase daily across the world. By 24 August 2020, South Africa (SA) was ranked fifth globally in the number of positive cases and had reported 611450 cases and 13159 deaths. ${ }^{[1]}$ The true number of deaths attributed to COVID-19 is likely to be higher than this. The South African Medical Research Council has been tracking mortality for decades in SA and estimated 36587 excess deaths in persons aged $\geq 1$ year by 11 August 2020 , compared with deaths in previous years..$^{[2]}$

According to estimates from countries in Europe and North America, ${ }^{[3,4]}$ up to half of those who died from COVID-19 were residents in long-term care facilities. In Europe it was realised some weeks into the pandemic that care facilities' data had not been routinely reported alongside statistics for COVID-19 deaths from hospitals. ${ }^{[5]}$ High-income countries were focused on hospitals at the start of the pandemic, lacked guidelines and protocols for care facilities, and were unprepared for 'catastrophic events' unfolding in care facilities that resulted in significant mortality. ${ }^{[3,6,7]}$

Residents of long-term care facilities are at risk for acquiring COVID-19 and for severe outcomes. COVID-19 appears to present an increased risk to people with neurocognitive deficits or intellectual and physical disabilities. ${ }^{[8]}$ The exact nature of the causal link is unclear at present and may be due to a variety of biological and environmental factors. Difficulties with adhering to frequent handwashing and physical distancing as well as poor insight and judgement regarding the risk of infection put them at increased risk of disease transmission. ${ }^{[6]}$ Being older and having multiple comorbidities is a leading indicator of poor outcomes from infection with COVID-19. ${ }^{[2]}$ In addition, the physical environment in long-term care facilities often requires performance of activities in communal spaces in close proximity that further complicates the management of outbreaks. ${ }^{[9]}$ Staff working in multiple homes with lack of training and inadequate personal protective equipment (PPE) have also been described as contributing factors. ${ }^{[2]}$ High risk for introduction of COVID-19 transmission in the care home setting includes: (i) residents hospitalised and then discharged back to the care home; and (ii) care home employees who may have acquired COVID-19 in the community.

Much work has already been done in SA to develop guidelines for preventing and managing COVID-19 in long-term care facilities, ${ }^{[10]}$ and to describe the impact of COVID-19 on long-term care facilities. ${ }^{[7]}$ In addition, the National Institute for Communicable Diseases (NICD) established an in-hospital surveillance platform on 1 April $2020,{ }^{[11,12]}$ which was expanded on 11 June 2020 to include surveillance for COVID-19 in care facilities.

There are estimated to be 1150 residential care homes for the elderly and 1000 private long-term care facilities for older persons in SA. ${ }^{[7]}$ The availability of long-term care facilities reflects urban-rural and historical racial divides, most are managed by non-governmental and faith-based organisations, and the standard of care in these facilities is highly variable. ${ }^{[7]}$ In collaboration with the national departments of Health and Social Development, a cohort of care facilities was identified to be enrolled across the country, to represent different types of care facilities in each province, in rural and urban settings, and in both affluent and impoverished communities.

In respect of the surveillance process, the data are submitted by an administrator or healthcare worker of the care facility into DATCOV, the NICD's online platform for COVID-19. Data are captured for both residents and care facility employees who test positive for COVID19, on demographics and comorbid conditions, and on outcomes (recovered, admitted to hospital or death). Deaths are reported on all individuals who had a COVID-19 laboratory-confirmed diagnosis and exclude deaths obviously attributed to other causes.

A preliminary analysis of data submitted by 19 long-term care facilities was conducted on 25 August 2020. These facilities included old-age homes and mental healthcare, rehabilitation and frail care facilities in Eastern Cape, Free State, Gauteng, Western Cape, Limpopo, Mpumalanga and KwaZulu-Natal provinces.

A combined total of 837 individuals tested positive for COVID-19. These included 502 residents (representing 10\% of the 4825 residents) and 335 staff (representing 12\% of the 2657 staff) in those facilities. The median (interquartile range) age of residents diagnosed with COVID-19 was $58(42-71)$ years and that of staff $42(35-50)$ years. There were 447 females reported as COVID-19 cases (53\%). Among the 770 cases with comorbid conditions (92\%), the most common were hypertension $(n=163 ; 21 \%)$ and diabetes $(n=60 ; 8 \%)$. Of the 502 residents, $375(75 \%)$ recovered, $53(10 \%)$ died and $74(15 \%)$ remained active cases as of 25 August 2020. Of the 335 staff, 318 (95\%) recovered, $4(1 \%)$ died and $13(4 \%)$ remained active cases as of the same date.

The residents and staff of care facilities are a vulnerable group in the COVID-19 pandemic, and surveillance has revealed that $10 \%$ of residents and $12 \%$ of staff have acquired the disease in 19 facilities. Expanding the NICD sentinel hospital surveillance for COVID-19 to a spectrum of care facility types has provided valuable information in high-risk groups around risk factors for disease and outcomes. This surveillance will serve to alert managers from the departments of Health and Social Development of possible outbreaks in care home settings, so that resources may be targeted where they are required and effective, co-ordinated responses from both departments can be ensured.

The following urgent recommendations are proposed around three key areas:

Infection prevention and control

- Develop comprehensive plans for infection prevention and control that include training and protocols around environmental cleaning and disinfection, personal hygiene and respiratory etiquette, PPE, waste disposal, staff travel, physical distancing, risk identification and family visits.

- Acquire essential equipment and resources such as PPE, digital thermometers, pulse oximeters, glucometers and medicine stocks.

- Determine advance human resources planning to ensure continuity of services.

\section{Early identification of cases}

- Test residents and staff who display symptoms and ensure rapid turnaround times $(<24$ hours) for laboratory results. (Owing to low test capacity and long turnaround times, regular testing for asymptomatic individuals without known or suspected SARSCoV-2 exposure for early identification in special settings is not feasible at this time.)

- Report positive cases to authorities, including the NICD care home surveillance system.

- Postmortem confirmatory testing for suspected COVID-19 should be sought wherever possible to determine the cause of death in residents. 
Clinical management and early referral where required

- Review of the facility structure with creation of isolation and quarantine 'zone' spaces or wards.

- Early isolation of positive cases and quarantine of close contacts.

- Ensure sufficient supplies of chronic medications for residents with comorbid conditions.

- Ensure that residents have basic monitoring of glucose, blood pressure and other measures, and prompt clinical management if they are confirmed with COVID-19.

- Ensure that referral systems are known in advance, as patients can decompensate rapidly and may need to be transferred to hospital to receive the required medical or surgical attention.

- Determine advance care plans and palliative care requirements and maintain ongoing communication with families. Advance care planning is a process that enables individuals to make plans about their future healthcare. Advance care plans provide direction to healthcare professionals when a person is not in a position to make and/or communicate their own healthcare choices.

- Support residents and staff within the facilities to manage stress.

It is not too late to recognise the risks in long-term care facilities and to provide these vulnerable settings with the support and resources that they require.

\section{Beverley Cowper, Waasila Jassat}

Division of Public Health Surveillance and Response, National

Institute for Communicable Diseases of the National Health

Laboratory Service, Johannesburg, South Africa

beverley.cowper@sanofi.com

\section{Paul Pretorius}

Professor and Chief Specialist: Department of Psychiatry, Faculty of Health Sciences, University of the Free State, Bloemfontein, South Africa

\section{Leon Geffen}

Executive Director: SIFAR (Samson Institute for Ageing Research); and Honorary Senior Lecturer, Institute of Ageing in Africa, University of Cape Town, South Africa

\section{Civil Legodu}

Chief Director: Professional Support and Older Persons, National Department of Social Development, Pretoria, South Africa

\section{Sandhya Singh}

Director: Non-Communicable Diseases, National Department of Health, Pretoria, South Africa

\section{Lucille Blumberg}

Deputy-Director: Epidemiology and Medical Consultant, Centre for Emerging and Zoonotic Diseases, National Institute for Communicable Diseases of the National Health Laboratory Service, Johannesburg, South Africa

1. Johns Hopkins University. COVID-19 dashboard by the Centre for Systems Science and Engineering. https://gisanddata.maps.arcgis.com/

2. Bradshaw D, Laubscher R, Dorrington RE, Groenewald P, Moultrie T. Report on weekly deaths in South Africa, 1 January - 11 August 2020. South African Medical Research Council, 2020. https://
Sadsing www.samrc.ac.za/reports/report-weekly-deaths-south-africa (accessed 25 August 2020).

3. Declercq A, de Stampa M, Geffen L, et al. Why, in almost all countries, was residential care for older people so badly affected by COVID-19? OSE Working Paper Series, Opinion Paper No. 23. European Social Observatory, Brussels, July 2020. http://www.ose.be/files/publication/OSEPaperSeries/ Declercq_Et_Al_2020_OpinionPaper23.pdf (accessed 18 July 2020).

4. Béland D, Marier P. COVID-19 and long-term care policy for older people in Canada. J Aging Soc Policy 2020;32(4-5):358-364. https://doi.org/10.1080/08959420.2020.1764319

5. Iacobucci G. Covid-19: Lack of testing led to patients being discharged to care homes with virus, say auditors. BMJ 2020;369:m2375. https://doi.org/10.1136/bmj.m2375

6. British Geriatrics Society. Coronavirus and older people. https://www.bgs.org.uk/resources/ resource-series/coronavirus-and-older-people (accessed 18 July 2020).

7. Ashwell A, Jacobs R, Docrat S, Schneider M. The impact of Covid-19 on long-term care facilities in South Africa with a specific focus on dementia care. STRiDE South Africa, last updated 10 July 2020. http://sifar.org.za/sites/default/files/field/file/SA\%20LTCCovid\%201st\%20interim\%20 report_10\%20July\%202020_Appendix\%20added.pdf (accessed 18 July 2020).

8. Turk M, Landes S, Formica M, Goss K. Intellectual and developmental disability and COVID-19 case-fatality trends: TriNetX analysis. Disabil Health J 2020;13(3):100942. https://doi.org/10.1016/j. dhjo.2020.100942

9. Xiang YT, Zhao YJ, Liu ZH, et al. The COVID-19 outbreak and psychiatric hospitals in China: Managing challenges through mental health service reform. Int J Biol Sci 2020;16(10):1741-1744. https://doi.org/10.7150/ijbs.45072

10. Kelly G, Geffen L. Guidelines, standard operating procedures and clinical practice - preventing and managing COVID-19 infections in long-term care facilities. SIFAR (Samson Institute for Ageing Research), 25 June 2020. https://www.sifar.org.za/sites/default/files/field/file/SOPS\%20Version\%20 $2 \% 2025 \% 20 J u n e . p d f$ (accessed 18 July 2020).

11. Blumberg L, Jassat W, Mendelson M, Cohen C. The COVID-19 crisis in South Africa: Protecting the vulnerable. S Afr Med J 2020;110(9):823-824. https://doi.org/10.7196/SAMJ.2020.v1 10i9.15116

12. Jassat W, Cohen C, Kufa T, et al. DATCOV: A sentinel surveillance programme for hospitalised individuals with COVID-19 in South Africa, 2020. COVID-19 Special Public Health Surveillance Bulletin, Vol. 18, Supplementary Issue 1. National Institute for Communicable Diseases, 10 June 2020. https://www.nicd.ac.za/wp-content/uploads/2020/06/COVID-19-Special-Public-HealthSurveillance-Bulletin-10-June-2020-005.pdf (accessed 8 July 2020).

S Afr Med J 2020;110(10):962-963. https://doi.org/10.7196/SAMJ.2020.v110i10.15214 